\title{
Contrast-enhanced ultrasonography
}

\author{
Wesley D. Block MD PhD, Mostafa Atri MD
}

\section{Contrast-enhanced ultrasonography uses microbubble contrast agents}

Microbubble contrast agents have a gaseous core, such as perfluorocarbon, surrounded by a biocompatible shell that prevents gas leakage and aggregation. The contrast agent is administered intravenously. Reflected sound waves, or echoes, from the microbubbles are distinguished from tissue echoes (using techniques such as pulse inversion) and are used to produce images in which intensity is proportional to vascularity and tissue perfusion.

\author{
Microbubbles do not infiltrate \\ interstitial spaces
}

This characteristic of microbubbles potentially makes them ideal for assessing blood flow volumes within lesions, for example when monitoring tumour response to therapy. Thus, contrast-enhanced ultrasonography is more sensitive than contrastenhanced computed tomography (CT) or magnetic resonance imaging (MRI) in detecting low perfusion in both normal and abnormal tissues. ${ }^{1}$

\section{Characterization of liver masses is the most robust indication}

In a retrospective review of more than 1000 patients, contrast-enhanced ultrasonography was $89 \%$ accurate in characterizing liver masses. ${ }^{2}$ In assessing focal liver lesions diagnosed using conventional imaging criteria, the technique had a sensitivity of $92.0 \%$ and a specificity of $86.7 \%$ for hepatocellular carcinoma $(n=$ $87)$, and a sensitivity of $96.3 \%$ and specificity of $97.5 \%$ for hemangiomas $(n=$ 26). ${ }^{3}$ An example of this technique for biopsy-proven hepatocellular carcinoma is shown in Figure 1.
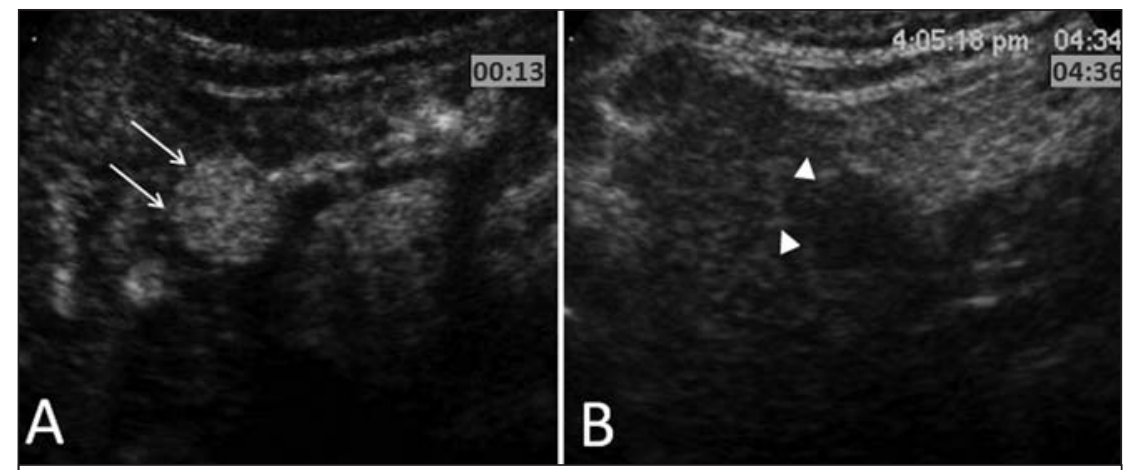

Figure 1: Contrast-enhanced ultrasound images of biopsy-proven hepatocellular carcinoma. (A) Axial image through the liver in the arterial phase (13 s) shows a round lesion with marked enhancement (arrows) relative to surrounding liver parenchyma. (B) Corresponding delayed-phase image shows decreased enhancement within the lesion (arrowheads); this washout sign is characteristic of malignant disease.

Contrast-enhanced ultrasonography is useful in assessing renal and abdominal masses

Established roles for this technique include assessing renal masses with indeterminate enhancement on CT imaging, evaluating the vascularity of abdominal masses, and assessing for leaks after repair of endovascular aneurysms in patients with a contraindication to CT or MRI, such as an allergy to contrast agents or impaired renal function. ${ }^{1}$

Microbubbles have an excellent safety profile

Microbubbles are approved for clinical use in more than 50 countries, and more than three million injections have been administered. Anaphylactoid reaction is the most frequent severe adverse event, occurring in $0.014 \%$ of patients. ${ }^{4,5}$ This frequency is similar to that of such reactions to antibiotics and analgesics. ${ }^{6}$ Unlike CT or MRI contrast agents, microbubbles can be used regardless of the patient's renal function.

For references, please see Appendix 1, available at www.cmaj.ca/lookup/suppl /doi:10.1503/cmaj.112057/-/DC1

Competing interests: None declared.

This article has been peer reviewed.

Affiliations: From the Department of Medical Imaging, Faculty of Medicine, University of Toronto, Toronto, Ont.

Correspondence to: Dr. Mostafa Atri, mostafa.atri@uhn.ca

CMAJ 2012. DOI:10.1503/cmaj.112057 